\title{
A Fuzzy Approach To Classify Learning Disability
}

\author{
Pooja Manghirmalani \\ Department of Computer Science \\ University of Mumbai \\ Mumbai-98, India.
}

\author{
Darshana More \\ Department of Computer Science \\ University of Mumbai \\ Mumbai-98, India.
}

\author{
Kavita Jain \\ Department of Computer Science \\ University of Mumbai \\ Mumbai-98, India.
}

\begin{abstract}
The endeavor of this work is to support the special education community in their quest to be with the mainstream. The initial segment of the paper gives an exhaustive study of the different mechanisms of diagnosing learning disability. After diagnosis of learning disability the further classification of learning disability that is dyslexia, dysgraphia or dyscalculia are fuzzy. Hence the paper proposes a model based on Fuzzy Expert System which enables the classification of learning disability into its various types. This expert system facilitates in simulating conditions which are otherwise imprecisely defined.
\end{abstract}

Keywords- Learning Disability; Dyslexia; Dysgraphia; Dyscalculia; Diagnosis; Classification; Fuzzy Expert System.

\section{INTRODUCTION}

Learning disability refers to a neurobiological disorder which affects a person's brain and interferes with a person's ability to think and remember [1]. The causes that lead to learning disability (LD) are maturational delay, some unexplained disorder of the nervous system and injuries before birth or in early childhood. Children born prematurely and children who had medical problems soon after birth can also inherit LD[2].LD can be broadly classified into three types. They are difficulties in learning with respect to read (Dyslexia), to write (Dysgraphia) or to do simple mathematical calculations (Dyscalculia) [3].

The term "specific learning disability" means a disorder in one or more of the basic psychological processes involved inunderstanding or in using language, spoken or written, which may manifestitself in an imperfect ability to listen, speak, read, write, spell, or to do mathematical calculations.The term includes such conditions as perceptual handicaps, brain injury, minimal braindysfunction, dyslexia, and developmentalaphasia. The term does not include children who havelearning disabilities which are primarily the result of visual, hearing, or motor handicaps, ormental retardation, or emotional disturbance, or of environmental, cultural, or economicdisadvantage [4].

A child has a specific learning disability if a severe difference between achievement and intellectual ability in one or more of the areas: (1) oral expression; (2) listening comprehension; (3) written expression; (4) basic reading skill; (5) reading comprehension; (6) mathematics calculation; or (7) mathematic reasoning [5].

LD cannot be cured completely by medication. Children suffering from LD are made to go through a remedial study in order to make them cope up with non-LD children of their age. For detecting LD, there does not exist a global method.

This paper proposes a model for diagnosis and classification of LD. Section II of this paper explores in detail different computational methods and models applied in detecting or diagnosing LD. Having elaborately explored different approaches, we have found that there are still possible ways of approaching the given problem. Section III discusses the Fuzzy Expert System designed to classify the problem of LD. Section IV gives the implementation requirement of the system and sections $\mathrm{V}$ and VI discusses the results and conclusion respectively.

\section{TAXONOMY}

The different computational methods and models used in detecting LD can be classified into four groups. The grouping is done based on the broader theoretical foundation and computational characteristics of the models and methods applied. The following sub- section deals with such models and their accuracy to diagnose LD. Once a child is diagnosed with $\mathrm{LD}$, the classification of LD can be done. The literature discusses various methodologies likeDigital Signal Processing (DSP), Digital Imageprocessing (DIP), Soft Computing and hybrid form ofthese techniques to diagnose LD.

Reitano [6] used DSP techniques to diagnose LD by comparing pre-recorded and properly pronouncedphonemes with mispronounced phonemes. Fonseca et al[7] conducted electroencephalograms (EEG) to detectabnormalities related to electrical activity of the brain bystudying different brainwaves. He concluded that there isa significant difference in brainwaves of normal andlearning disabled children.

Mico-Tormos et al [8] inferred that eye movements ofeven an infant could indicate LD by analyzing theresponses of the improvement of eye through Oculographic signals. Pavlidis [9] observed that erratic and strikinglylarge number of regressive eye movements pointed todyslexia.

Jain et al [10] proposed a simple perceptron basedartificial neural network (ANN) model for diagnosing LDusing curriculum based test conducted by specialeducators. Bullinaria [11] applied a multi-layer feedforward perceptron to diagnose dyslexia where letterstrings were mapped to phoneme strings in multi- syllabicwords. $\mathrm{Wu}$ et al [12] proved that multi-layer perceptron withback propagation gave better results in diagnosing LD. Helater attempted to diagnose LD using support vectormachines (SVM) [13]. Salhi et al [14] used both wavelettransforms and ANN to diagnose LD from 
pathologicalvoices. Novak et al [15] have calculated a set of featuresfrom signals of horizontal and vertical eye movement using self-organizing map and genetic algorithm (GA). They concluded that the reading speed increased with the probability of the patient being healthy.

Wu et al [16] further combined different feature selection algorithms like brute-force, greedy and GA along with ANN to improve the identification rate of LD. Georgopoulos et al [17] proposed that a hybridization of GA and fuzzy cognitive map was better equipped for accurate diagnosis. Macašet al [18] developed a system for extracting the features of eye movements from time and frequency domain. They concluded that back propagation based classification gave better results than that offered by Bayes' and Kohonen network.

Manghirmalani et al proposed a soft computing technique called Learning Vector Quantization. The model classifies a child as learning disabled or non- learning disabled. Once diagnosed with learning disability, rule based approach is used further to classify them into types of learning disability that is dyslexia, dysgraphia and dyscalculia [19].

Given in Table-1 is the comparative study of different soft computing techniques based on their accuracy.

TABLE I. COMPARATIVE STUDY OF DIFFERENT SOFT COMPUTING MODELSDESIGNED TO DIAGNOSE LD.

\begin{tabular}{|l|c|}
\hline \multicolumn{1}{|c|}{ Soft Computing Methodology } & Accuracy in \% \\
\hline Classification Tree & $71.79 \%$ \\
\hline K Nearest Neighbour Algorithm & $76.92 \%$ \\
\hline Simple Vector Machine Algorithm & $80.95 \%$ \\
\hline Bayes Classifier & $82.69 \%$ \\
\hline Single Layer Perceptron Algorithm & $84.00 \%$ \\
\hline $\begin{array}{l}\text { Feature Selection of Multilayer Perceptron } \\
\text { Algorithm }\end{array}$ & $86.00 \%$ \\
\hline Back Propagation Algorithm & $86.54 \%$ \\
\hline Logistic Regression Algorithm & $87.18 \%$ \\
\hline $\begin{array}{l}\text { Multilayer Perceptron Algorithm with } \\
\text { Genetic Algorithm }\end{array}$ & $88.2 \%$ \\
\hline $\begin{array}{l}\text { Back-Propagation } \\
\text { Learning Algorithm }\end{array}$ & $94.23 \%$ \\
\hline $\begin{array}{l}\text { Multilayer Perceptron Algorithm } \\
\text { Naive Bayes classifier }\end{array}$ & $91.8 \%$ \\
\hline Decision Tree classifier & \\
\hline Learning Vector Quantization Algorithm \\
\hline
\end{tabular}

\section{SOFT COMPUTING APPROACH - FUZZY EXPERT SYSTEM FOR CLASSIFYING LEARNING DISABILITY}

The soft computing technique called Fuzzy Expert System provides an alternative way to model human thinking with the concept of linguistic variables [20]. It deals with real world vagueness that isthe observation of parent and teacher. Hence classifying LD using this approach is quite significant.

\section{A. Collection of Exhaustive Parameters}

A curriculum-based test was designed with respect to the syllabus of primary-level school going children. This test was conducted in schools for collecting LD datasets for testing. Historic data for LD cases were collected from LD Clinics of Government hospitals where the tests were conducted in realtime medical environments. The system was fed with 11 input units which correspond to 11 different sections of the curriculum-based test.

Table-1 shows the initial 11 inputs corresponding to curriculum-based test. Column 1 represents the name of the parameter, column-2 represents the total marks allocated to a particular section, and column-3 determines the category of LD a section corresponds to.

Dataset consists of 170 cases of LD children. The system was trained using 100 data items and the remaining was used to test the system.

TABLE II. PARAMETERS AND MARKS OF CURRICULUM -BASED TEST.

\begin{tabular}{|c|c|c|}
\hline Input Parameter & Marks & Category of LD \\
\hline Essay & 10 & Dysgraphia \\
\hline Reading & 10 & Dyslexia \\
\hline Comprehension & 10 & Dyslexia,Dysgraphia \\
\hline Spelling & 10 & Dysgraphia \\
\hline Perception & 10 & Dyslexia \\
\hline Solve & 10 & Dysgraphia \\
\hline Word Problem & 10 & Dyscalculia,Dyslexia \\
\hline Mental Sums & 10 & Dyscalculia \\
\hline Time & 10 & Dyscalculia \\
\hline Calander & 05 & Dyscalculia \\
\hline Money & 05 & Dyscalculia \\
\hline
\end{tabular}

\section{B. Fuzzy Expert System for LD}

The literature survey indicates that most of the work done till now are for diagnosing the problem i.e. diagnosing methods can only detect whether a child is having or not having an LD, which is anbinary output.

The model proposed below assists in the classification oftype of LD that is dyslexia, dysgraphia or dyscalculia. Since fuzzy expert system is the best method for classification in soft computing, it is used in this system which can give an accuracy of approximately $90 \%$. 
Fuzzy expert system is able to process incomplete data and provide approximate solutions to problems which other methods find difficult to solve. Also fuzzy expert system uses rules which are the most fundamental part for classification purpose.

The reasoning in fuzzy expert system is similar to human reasoning. It allows for approximate values and inferences as well as incomplete or ambiguous data (fuzzy data) as opposed to only relying on crisp data.

Terminologies used in fuzzy expert system are not used in other methods [21]. They are: very high, increasing, somewhat decreased, reasonable and very low.

\section{a) A Fuzzy Logic System}

A fuzzy logic system (FLS) can be defined as the nonlinear mapping of an input data set to a scalar output data. A FLS consists of four main parts: fuzzifier, rules, inference engine, and defuzzifier. FLS is one of the most famous applications of fuzzy logic and fuzzy sets theory. They can be helpful to achieve classification tasks, offline process simulation and diagnosis, online decision support tools and process control[22].

As shown in figure-1, firstly, a crisp set of input data are gathered and converted to a fuzzy set using fuzzy linguistic variables, fuzzy linguistic terms and membership functions. This step is known as fuzzification. Afterwards, an inference is made based on a set of rules. And lastly, the resulting fuzzy output is mapped to a crisp output using the membership functions, in the defuzzification step.

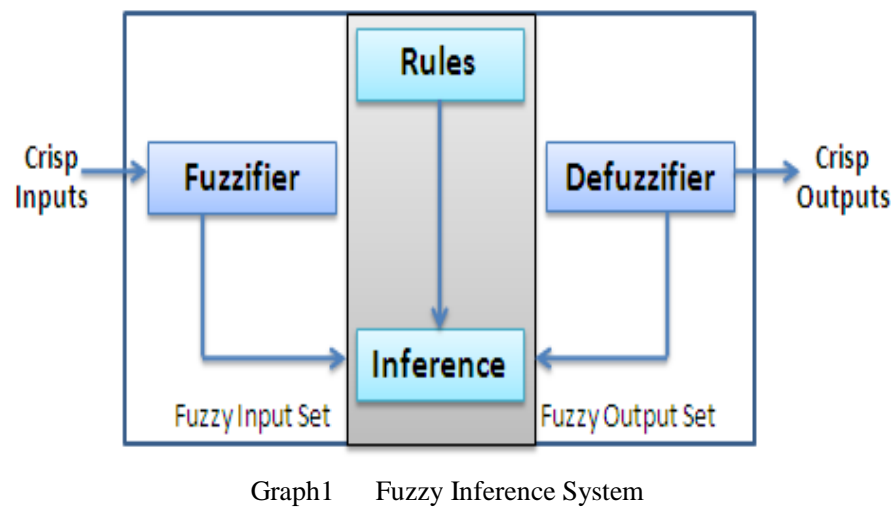

\section{b) Fuzzy Modeling}

As show in the figure-2, given below are the seven steps of fuzzy model applied to the data [23].

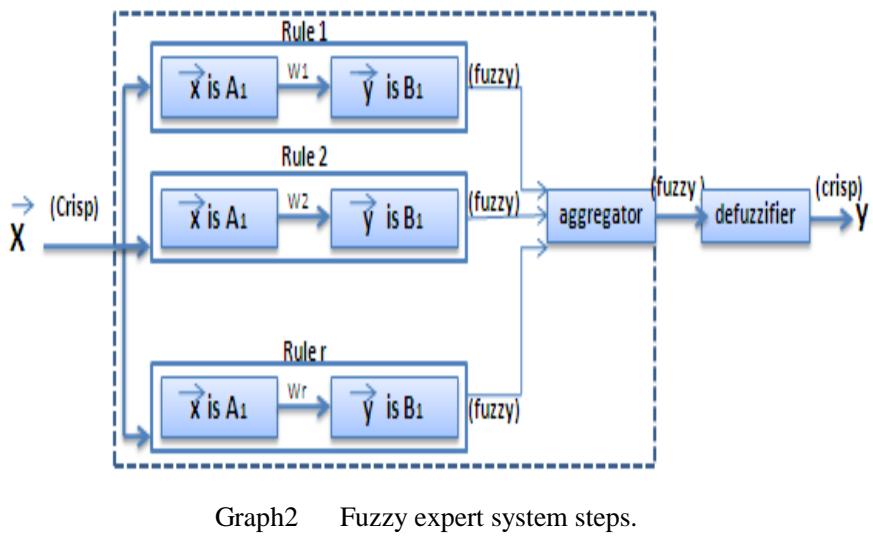

1. Define the linguistic variables and terms.

2. Construct the membership functions.

3. Construct the rule base.

4. Convert crisp input data to fuzzy values using the membership functions.

5. Applying implication method.

6. Aggregating all output.

7. Convert the output data to non-fuzzy values.

1) Lingusistic variables

- The first step is to take inputs and determine the degree to which they belong to each of the appropriate fuzzy sets via membership functions.

- $\quad$ Since there are 11 inputs out of which 9 are from 0 to 10 and 2 are from 0 to 5 . For more classification ofthese inputs are partitioned into 4 linguistic variables Poor, Satisfactory, Good and Excellent.

- Applying Formula for each of these linguistic variables, 4 values in the range of 0 to 1 are returned respectively. Calculating the membership function values all are stored into database.

2) Formula for Membership Function

If variable as "input" is taken for the inputs of the system, then: [23] 
a) for linguistic variables "Poor"

$$
\begin{array}{ll}
\text { memfunc }=1.0 ; & \text { if--input }>=0 \text { \& \& input }<=4 \\
\text { memfunc }=(4.5 \text {-input }) / 0.5 & \text { if--input }>4 \& \& \text { input }<=4.5 \\
\text { memfunc }=0.0 & \text { if--input }>4.5 \& \& \text { input }<=10
\end{array}
$$

b) for linguistic variables "Satisfactory"

$$
\begin{array}{ll}
\text { memfunc }=0.0 ; & \text { if--input }>=0 \& \& \text { input }<=4 \\
\text { memfunc }=(\text { input-4 }) / 0.5 & \text { if--input }>4 \& \& \text { input }<=4.5 \\
\text { memfunc }=1.0 & \text { if-- input }>4.5 \& \& \text { input }<=5.5 \\
\text { memfunc }=(6 \text {-input }) / 0.5 & \text { if-- input }>5.5 \& \& \text { input }<=6 \\
\text { memfunc }=0.0 & \text { input }>6 \& \& \text { input }<=10
\end{array}
$$

c) for linguistic variables "Good"

$$
\begin{array}{ll}
\text { memfunc }=0.0 ; & \text { if--input }>=0 \& \& \text { input }<=5.5 \\
\text { memfunc }=(\text { input }-5.5) / 0.5 & \text { if--input }>5.5 \& \& \text { input }<=6 \\
\text { memfunc }=1.0 & \text { if-- input }>6 \& \& \text { input }<=7 \\
\text { memfunc }=(7.5 \text {-input }) / 0.5 & \text { if-- input }>7 \& \& \text { input }<=7.5 \\
\text { memfunc }=0.0 & \text { input }>=7.5 \& \& \text { input }<=10
\end{array}
$$

d) for linguistic variables "Excellent"

$$
\begin{array}{ll}
\text { memfunc }=0.0 ; & \text { if--input }>=0 \& \& \text { input }<=7 \\
\text { memfunc }=(\text { input-7)/0.5 } & \text { if--input }>7 \& \& \text { input }<=7.5 \\
\text { memfunc }=1.0 & \text { if--input }>7.5 \& \& \text { input }<=10
\end{array}
$$

In this manner, each input is fuzzified over all the qualifying membership functions required by the rules.

3) Rules of Classification

Case-1: IF score low in fields of essay, reading, comprehension, spelling, perception, solve, word problems, mental sums, time, money;

THEN Dyslexia, Dysgraphia and Dyscalculia

Case-2: IF score low in fields of reading, comprehension, perception, word problem;

\section{THEN Dyslexia}

Case-3:IF score low in fields of spelling, comprehension, essay;

\section{THEN Dysgraphia}

Case-4:IF score low in fields of solve, mental sums, word problems, sums related to time, calendar, money;

THEN Dyscalculia

Case-5: IF score low in fields of reading, comprehension, perception word problem, spelling, essay;

THEN Dyslexia and Dysgraphia

Case-6: IF score low in fields of reading, comprehension, perception, solve, mental sums, word problems, sums related to time, calendar and money;

THEN Dyslexia and Dyscalculia
Case-7: IF score low in fields of spelling, comprehension essay, solve, mental sums, word problems, sums related to time, calendar,money;

THEN Dysgraphia and Dyscalculia

Many a times, one type of LD leads to other two types as well; thus most children are detected with all three types of LDs. Unique cases have one type of LD diagnosed or sometimes a combination of two [24].

1) Fuzzy Operators

- Now here the Rules are applied which have two parts[23]:

\section{$>$ Antecedent (before "Then") \\ $>$ Consequent (after "Then").}

- Once the inputs have been fuzzified, we know the degree to which each part of the antecedent. The values which are stored into the database are fetched for applying rules. By following the rules, the "Poor" linguistic variable is taken into consideration.

- For the 2nd rule, fields of reading, comprehension, perception, word problem; where the poor scores of these fields are taken and the AND(MIN) operator is applied to the linguistic values of each field.

- $\quad$ For Eg. Suppose Rule 2 has values like $\{0.2,0.7,0.8$, $0.1\}$ thus the output will be 0.1 . Thus forming a set, for all students, let us consider this set as the 1st array.

2) Applying Implication Method

- The input for the implication process is a single number given by the antecedent, and the output is a fuzzy set. Implication is implemented for each rule.

- Two built-in methods are supported, and they are the same functions that are used by the AND method: min (minimum), which truncates the output fuzzyset, and prod (product), which scales the output fuzzy set.

- As the membership values are calculated for antecedents, the membership functions for the consequents are also calculated. Thus a set of values for each input and thus for all the students is computed. Let us consider this set as the 2nd array.

- The implication operator is applied i.e. the Min Operator between the values of the antecedent and the consequent. Thus a fuzzy set is computed for all students, and for each rule fuzzy sets are formed.

- Implication method finds the minimum of the corresponding values from the 1st array and the 2nd array thus forming a new set

- For Eg. The value of 1 st array is 0.1 and 2 nd array is 0.4 , then the output of implication method will be 0.1 . Each rule will give this kind of values, to form sets.

3) Aggregrating all Outputs 
- Because decisions are based on the testing of all of the rules in a FIS, the rules must be combined in some manner in order to make a decision.

- Aggregation is the process by which the fuzzy sets that represent the outputs of each rule are combined into a single fuzzy set.

- As long as the aggregation method is commutative (which it always should be), then the order in which the rules are executed is unimportant. Three built-in methods are supported[23]:

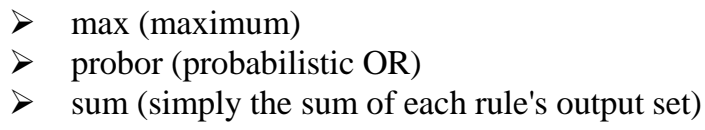

- The rules are combined using the Sum aggregation method which is formed into a single fuzzy set.

- Since there are $3 \mathrm{LDs}$, the rules output sets are aggregated to form 3 Aggregation sets, which can be further defuzzified.

\section{4) Defuzzify}

- The input for the defuzzification process is a fuzzy set (the aggregate output fuzzy set) and the output is a single number.

- There are five built-in methods supported: centroid, bisector, mean of maximum (the average of the maximum value of the output set), largest of maximum, and smallest of maximum[23].

- Perhaps the most popular defuzzification method is the Centroid calculation, which returns the center of area under the curve.

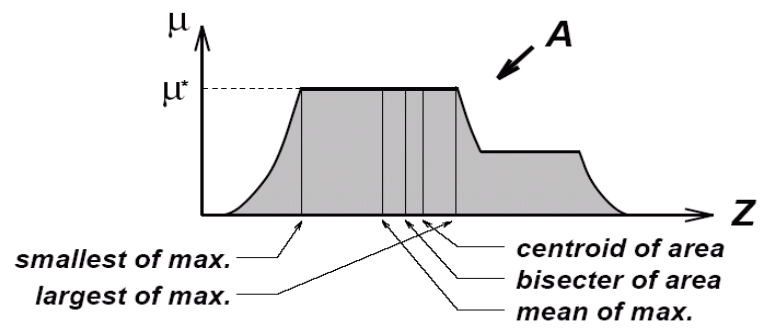

Graph3 Different methods for Defuzzification

- Centroid Defuzzification formula[27]:

$$
z_{C O A}=\frac{\int_{Z} \mu_{A}(z) z d z}{\int_{Z} \mu_{A}(z) d z},
$$

where $\mu_{\mathrm{A}}(\mathrm{z})$ is the value from the aggregated set and $\mathrm{z}$ is the range of value from 0 to 100 .

- Example: If one of the aggregated set contains values

$$
\{0.2,0.5,0.8,1\}
$$

thenthe defuzzified value is equal to :

$$
=(10+20+30+40) 0.2+(50+60+70) 0.5+(80+90) 0.8+(100) 1
$$

$(0.2+0.2+0.2+0.2+0.5+0.5+0.5+0.8+0.8+1)$

$$
=72.24
$$

Thus, by using this formula, the threshold values for all 3 LDs, by performing 3 defuzzifications.

c) Classification for $L D$

- After some processing, at the aggregation step the rules need to be aggregated.

- While aggregation the classification is done on the basis that the rules having dyslexia as their consequent are aggregated together. (Same is done for dysgraphia and dyscalculia.)

- Thus, the aggregations are defuzzified for all three LDs and thus getting the output which in the form of percentage.

- This value being the required threshold value is used for proper classification.

- Example: If the value getting after inputting new data, is less than or equal to the threshold value, then he/she is suffering from that particular LD.

\section{IMPLEMENTATION}

The system is implemented using JAVA. The data collected is stored in Excel sheets. The experiments were conducted on a workstation with an Intel Pentium(R) 4 CPU, 3.06ghz, 2GB of RAM, running on Microsoft Windows XP Home Edition, Version 2002 with Service Pack 3.

\section{RESUltS}

\section{A. Classification for Correct Type}

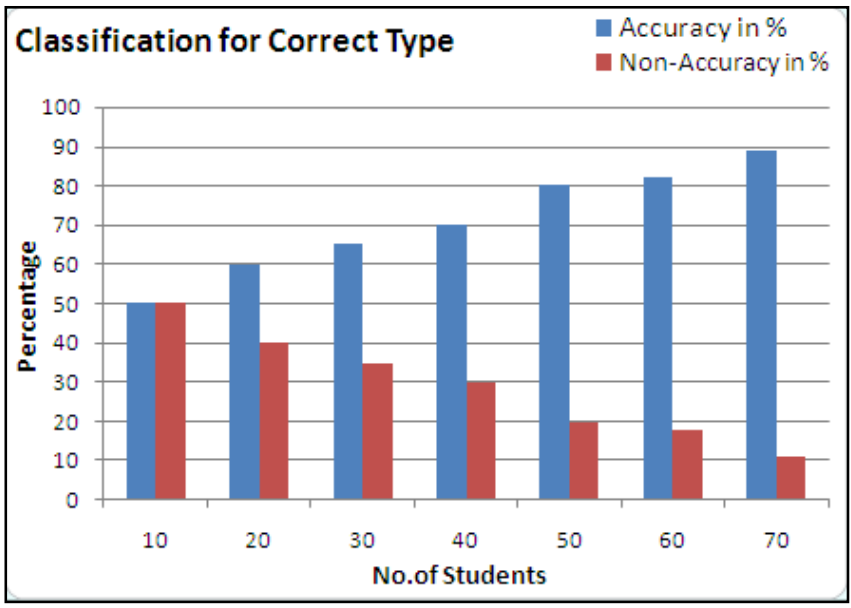

Graph4 Classification for Correct type of LD showing the accuracy and the non- accuracy during the training phase.

Graph-1 gives an overview of the system's accuracy rate. As more data is added to the database, the system's accuracy increases.Initially when the database had 10 records as Training Set, it gave an accuracy of $50 \%$, i.e. the system correctly classified Learning Disabilities 50\% accurately. When data in the system training set was increased to 20, the accuracy rate went up to $60 \%$ and so on.For 70 data in the Training set, the system is giving an accuracy of $90 \%$. 


\section{B. Classification for Specific $L D$}

Graph-2 shows that, after all training and testing of the system, the percentage for all 3 types of LDs is $90 \%$.

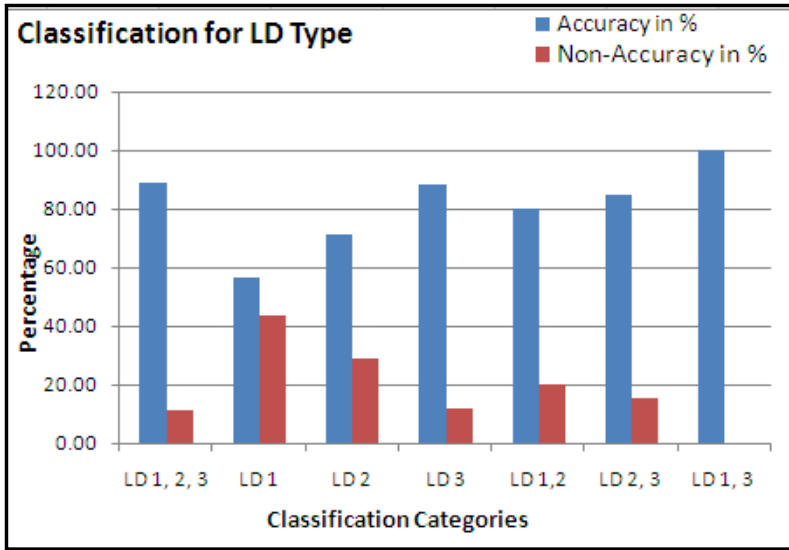

Graph5 Graph 2: Classification for specific LD showing the accuracy and the non-accuracy during the training phase.

\section{CONCLUSION AND FUTURE WORK}

After never-ending deliberations with doctors, special educators, teachers and parents of children with LD, we could understand and monitor the process of classifying LD in India. Soft computing and other techniques are helpful in diagnosing LD with great accuracy, but once diagnosed, it is still difficult to judge the specific LD amongst children. Thus the remedial education given to these cases becomes very general.

We have made an attempt to classify LD using yet another soft computing technique called Fuzzy Expert System. The method is not only simple and easy toreplicate in huge volumes but gives good resultsbased on accepted benchmarks. However, there is scope for further enhancement of system by finding a combination of algorithms so as to build up a model that is satisfactorily more accurate. It is also seen that on increasing the number of data in the training set of the system, the overall accuracy shows a promising growth.

In future we intend to explore the possibility of parameter classification in order to distinguish irrelevant and superfluous variables which might lead to decrease in diagnosis process time and increase in accuracy. This can be beneficial for the special educators, doctors and teachers by providing suggestions that lead to the exclusion of redundant tests and saving of time needed for diagnosing LD. On the whole, the focus of our research is to identify early diagnosis and proper classification of LD and to support special education community in their quest to be with mainstream.

\section{ACKNOWLEDGMENT}

Authors acknowledge the contributions of accredited Government hospitals and schools in Mumbai, India. They also express their gratitude to doctors, special educators and parents of LD diagnosed children for their time, support, guidance and patience.

\section{REFERENCES}

[1]. S.A Kirk, Educating Exceptional Children Book, Wadsworth Publishing, ISBN: 0547124139.
[2]. Lisa L. Weyandt; "The physiological bases of cognitive and behavioral disorders"; Blausen Medical Communications, United States.

[3]. Lerner, Janet W, "Learning disabilities: theories, diagnosis, and teaching strategies"; Boston: Houghton Mifflin; ISBN 0395961149.

[4]. Fletcher, Francis,Rourke, Shaywitz \& Shaywitz; "Classification of learning disabilities: an evidencebased evaluation"; 1993.

[5]. Kaplan, Robert M.; Saccuzzo, Dennis P. "Psychological Testing: Principles, Applications, and Issues (Seventh ed.)", Belmot (CA): Wadsworth. p. 262 (citing Wechsler (1958) The Measurement and Appraisal of Adult Intelligence), ISBN 978-0- 495-09555-2, 2009.

[6]. Carmen T. Reitano, "System \& method for dyslexia detection by analyzing spoken \& written words", United States Patent US 6535853 B1 March 18, 2003

[7]. Lineu C. Fonseca, Glória M.A.S. Tedrus, Marcelo G.Chiodi, Jaciara Näf Cerqueira, Josiane M.F. Tonelotto Arq Neuropsiquiatr, "Quantitative EEG in children with learning disabilities", Analysis of band power, 64(2-B):376-381, 2006.

[8]. Pau Mico-Tormos, David Cuesta-Frau, Daniel Novak,"Early Dyslexia Detection Techniques by means of Oculographic Signals", 2nd European Medical \& Biological Engineering Conference, Vienna, Austria. December 2002.

[9]. George Th.Pavlidis, "Eye Movement in Dyslexia: Their Diagnostic Significance", Journal of Learning Disabilities, 2001.

[10]. Kavita Jain, Pooja Manghirmalani, Jyotshna Dongardive, Siby Abraham, "Computational Diagnosis of Learning Disability" , International Journal of Recent Trends in Engineering, Vol 2, No. 3, November 2009.

[11]. John A Bullinaria, "Neural Network Models of Reading MultiSyllabic Words", International Joint Conference on Neural Networks, 1993.

[12]. Tung-Kuang Wu, Ying-Ru Meng and Shian-Chang Huang, "Application of ANN to the identification of students with LD",IC-AI: 162-168. 9, 2006.

[13]. Tung-Kuang Wu, Ying-Ru Meng and Shian-Chang Huang, "Identifying \& Diagnosing Students with LD Using ANN \&SVM", IEEE International Joint Conference on Neural Networks, Vancouver, BC, 2006

[14]. L. Salhi, M. Talbi, A. Cherif, "Voice Disorders Identification usingHybrid Approach: Wavelet Analysis and Multilayer NeuralNetworks",Proceedings of World Academy of Science,Engineering and Technology Volume 35, ISSN 2070374,November 2008

[15]. D. Novak, P. Kordk, M. Macas, M.Vyhnalek, R. Brzezny, L.Lhotska "School Children Dyslexia Analysis using SelfOrganizing Maps",IEEE 26th Annual International Conference,San Francisco, CA, 2004.

[16]. Tung-Kuang Wu, Ying-Ru Meng and Shian-Chang Huang,"Effectsof Feature Selection on the Identification of Students with LDUsing ANN", Springer-Verlag Heidelberg, 2006.

[17]. Voula Georgopoulos and Chrysostomos Stylios, "GeneticAlgorithm enhanced fuzzy cognitive maps for medical diagnosis", IEEE International conference on Fuzzy Systems, Hong Kong,2008.

[18]. Martin Macaš, Lenka Lhotská and Daniel Novák, "Bioinspiredmethods for analysis and classification of reading eye movementsof dyslexic children", Department of Cybernetics,Czech TechnicalUniversity in Prague, Czech Republic NiSls Symposium 2005.

[19]. Pooja Manghirmalani, Zenobia Panthaky, Kavita Jain; 'Learning Disability Diagnosis and Classiication-a Soft Computing Approach'; IEEE World Congress on Information and Communication Technologies (WICT); DIO: 10.1109/WICT.2011.6141292, 2011.

[20]. R. Jang , T.Sun, E.Mizutani, Neuro -Fuzzy And Soft Computing

[21]. Novák, V., Perfilieva, I. and Močkoř, J. Mathematical principles of fuzzy logic Dodrecht: Kluwer Academic. ISBN 0-7923-8595-0, 1999.

[22]. M.A. Arjona L.,R. Escarela-Perez,E. Melgoza- 'Vázquezc, C. Hernández.F., Convergence improvement in two-dimensional finite element nonlinear magnetic problems - a fuzzy logic approach'; Finite Elements in Analysis and Design, Science Direct, 2004. DOI: 10.1016/S0168-874X(02)00165-8 
[23]. George J. Klir/Bo Yuan, "Fuzzy Sets and Fuzzy Logic: Theory and Applications"

[24]. J. Mendel. Fuzzy logic systems for engineering: a tutorial. Proceedings ofthe IEEE, 83(3):345\{377, Mar 1995

\section{AUTHORS PROFILE}

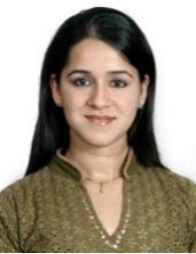

Pooja Manghirmalani: She has received her M.Sc. Computer Science degree from University of Mumbai. Currently she is working as an Assistant Professor at the Department of Computer Science, University of Mumbai, Mumbai, India. She has published papers in International Journals and conference proceedings for Learning Disability. Her research interest includes Artificial Intelligence, Machine Learning and Cloud Computing.

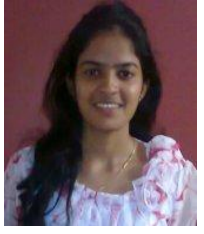

Darshana More: She is doing her M.Sc. Computer Science from University of Mumbai, Mumbai, India. She has worked on several government projects and has currently taken up research to support special children of the society. Her areas of interest include Artificial Intelligence, System \& Internet Security and Networking.

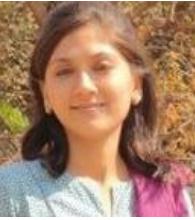

Kavita Jain: She has received her M.Sc. Computer Science degree from University of Mumbai. Currently she is working as an Assistant Professor at the Department of Computer Science, University of Mumbai, Mumbai, India. She has published papers in Internatinal Journals and Conference Proceedings for Learning Disability. Her research interest includes Bioinformatics, Artificial Intelligence and Machine Learning. 